\title{
Sensing Senses: Tactile Feedback for the Prevention of Decubitus Ulcers
}

\author{
Marcel Verbunt $\cdot$ Christoph Bartneck
}

Published online: 1 December 2009

(c) The Author(s) 2009. This article is published with open access at Springerlink.com

\begin{abstract}
Decubitus ulcers, also known as pressure sores, is a major problem in health care, in particular for patients with spinal cord injuries. These patients cannot feel the discomfort that would urge healthy people to change their posture. We describe a system that uses a sensor mat to detect problematic postures and provides tactile feedback to the user. The results of our preliminary study with healthy subjects show that the tactile feedback is a viable option to spoken feedback. We envision the system being used for rehabilitation games, but also for everyday Decubitus ulcers prevention.
\end{abstract}

Keywords Spinal cord injury - Tactile feedback · Decubitus ulcers prevention · Posture activity . Wheelchair

\section{Introduction}

Decubitus ulcers are an area of localized damage to the skin and underlying tissue caused by pressure, shear, friction and or a combination of these. In The Netherlands alone, the treatment of Decubitus ulcers cost 600 million euro per year, which is more than $1 \%$ of the total costs of healthcare in The Netherlands (Health Council of The Netherlands 1999). Braden and Bergstrom (1987) identified extrinsic and intrinsic factors that influence pressure sore development. Extrinsic factors include moisture, friction and shear.

M. Verbunt · C. Bartneck ( $\square)$

Department of Industrial Design, Eindhoven University

of Technology, Den Dolech 2, 5600 MB Eindhoven,

The Netherlands

e-mail: c.bartneck@tue.nl

M. Verbunt

e-mail: m.n.c.verbunt@student.tue.nl
Intrinsic factors include nutrition, age, arteriolar pressure and possibly also interstitial fluid flow, emotional stress, smoking and skin temperature. The risk of developing Decubitus ulcers is particularly high for people that have to spend most of their time in beds or wheelchairs because their skin is exposed to long durations of pressure. Within this group, people with a spinal cord injury (SCI) are amongst the most vulnerable. Besides being constraint to wheelchairs and beds, they also lack the ability to sense the pain that would urge healthy people to change their posture. Environmental aspects that cause irritation, such as moisture, are also not detected. Eighty-five percent of the people with SCI experience damages that are directly related to their inability to sense irritation or pain that would normally alert the human body (Revalidatiefonds 2009). Once Decubitus ulcers has developed the patient has to spend a very long time in a resting position to allow the damaged skin to heal. This decreases the patients' mobility even further, resulting in a reduction of their social life, which in turn can have an impact on their psychological well-being.

A typical scenario for the development of Decubitus ulcers is that the patient first receives training during rehabilitation. The patients undergo dramatic changes in their lifes and need to adjust to the new situation. Under the supervision of the nurses they are made aware of the necessity to frequently change their posture. However, once they return to their old environments, they often start to neglect changing their posture. It is difficult to pay attention to something one cannot perceive. Over time, their decreased posture activity leads to an increased risk of peak pressures and reduced variance in pressure relief (Merbitz et al. 1985; Stockton and Parker 2002). Hobson (1992) showed that the mean maximum pressures of patients with SCI are significantly higher compared to healthy people in all nine sitting postures he studied. 
The most common method of preventing Decubitus ulcers is to assess the risk of the patient with the Braden scale (Braden and Maklebust 2005) before selecting an appropriate prevention method. One of the most common prevention methods are pressure relieving pillows and mattresses. Five attributes of a pillow need to be taken into account (Sprigle and Schuch 1993): density (weight/volume ratio), stiffness (measure of softness), resilience (ability to recover shape), dampening (absorb impact loads) and envelopment (surface area covered). Based on these attributes the right pillow can be selected. A variety of materials and design are available, including generic countered foam, air filled pillows, water filled pillows, solid gel pillows, viscoelastic foam and segmented foam. The goal of all these pillows and mattresses is to distribute the weight of the patient equally. However, the pillows and mattresses remain completely passive. They cannot make the patients change their position.

In the hospital environment, first attempts for a more active bed date back to 1970s (Harvin and Hargest 1970; Roemer et al. 1975). The first automatic beds rotated the patient by turning the half-tube shaped bed. Nowadays the automatic bed is replaced by air-fluidized beds, which are an important and proven therapy for pressure sores. Patients rest on a bed of beadlike ceramic spherules through which filtered air is circulated, thereby simulating the mechanics of "fluid" movement (Strauss et al. 1991). This type of bed is used in extreme cases in which a wound has already developed. The more commonly used beds use alternating pressure mattresses. These mattresses alternate pressure on the body by changing the pressure of the different segments of the bed. The principles of these beds are useful for the healing process of Decubitus ulcers, but they are not practical in the every day wheel chair usage.

All the solutions presented above for the prevention and recovery from Decubitus ulcers have one thing in common: they neglect the potential of the user. In particular the potential of users that have a lower spinal cord injury (LSCI). These users are fully able to move their upper body. LSCI users between 18 and 40 years tend to have an active lifestyle. Their problem is not that they cannot move enough, but that they cannot sense when they should move their body. We therefore focused this project on giving these users back their lost sense. Once they are able to feel critical postures, they can move around and thereby decrease the risk of developing Decubitus ulcers. We also propose to introduce elements of game play into Decubitus ulcers prevention. The system should not be limited to being an alarm system, but also a platform for rehabilitation games, similar to how Nintendo's Wii is used in rehabilitation (Goldberg et al. 2008; Leder et al. 2008).

\section{Challenges}

This project faced two challenges: sensing critical postures and communicating them to the user. Several pressure measuring mats are already in the market, such as Tekscan's BPMS ${ }^{\mathrm{TM}}$ System, ${ }^{1}$ XSensor's $\mathrm{PX} 100^{2}$ and Vista Medical's FSA Seating Assessment. ${ }^{3}$ These mats measure the pressure between the body and the seating surface.

Naturally, the communication protocols of these commercial solutions are not openly available. We reverse engineered the communication protocol of the Vista Medical's FSA system to be able to receive the sensor readings of this pressure measuring mat. The real challenge was to define what sensor readings constitute a critical posture, because it has to take the posture of the user over time into account.

The second challenge was to find an appropriate communication channel to the user. The communication should be discreet, immediate, and easy to understand. We will now discuss these three requirements in more detail. We need to respect the privacy of the user and therefore it would be inappropriate to shout the behavior of the users out loud. Also, the communication should get the attention of the user immediately. It would be insufficient if the user notices a problem only after several minutes. Last, the communication should tell the user directly what the problem is and what the user should do next. Due to practical reasons, only the visual, auditory and tactile modalities are available for communication within this project. We will now quickly discuss their advantages and disadvantages.

The visual sense is the most powerful sense and it is possible to communicate a wealth of information through it. The commercial solutions listed above use screens to visualize the pressure distribution (see Fig. 1 for an example). Adding such a screen to the wheel chair would enable the user to see exactly where the problem is.

The main problem of visual feedback is that users have to look at it to notice the signal. Unless the screen would be positioned in the direct field of view, it would be difficult for the user to be aware of potential problems. Moreover, such a screen would obstruct the general vision and might therefore not be desirable.

Auditory feedback has the advantage of being able to directly grab the attention of the user, no matter where the user is looking at. It is therefore frequently used for alarms, such as fire alarms. This advantage is directly related to its

\footnotetext{
${ }_{1}$ Tekscan is located in South Boston, MA (http://www.tekscan.com/).

${ }^{2}$ XSensor is located in Calgary, Canada (http://www.xsensor.com/).

${ }^{3}$ Vista Medical is located in Winnipeg, Canada (http://www. pressuremapping.com/).
} 


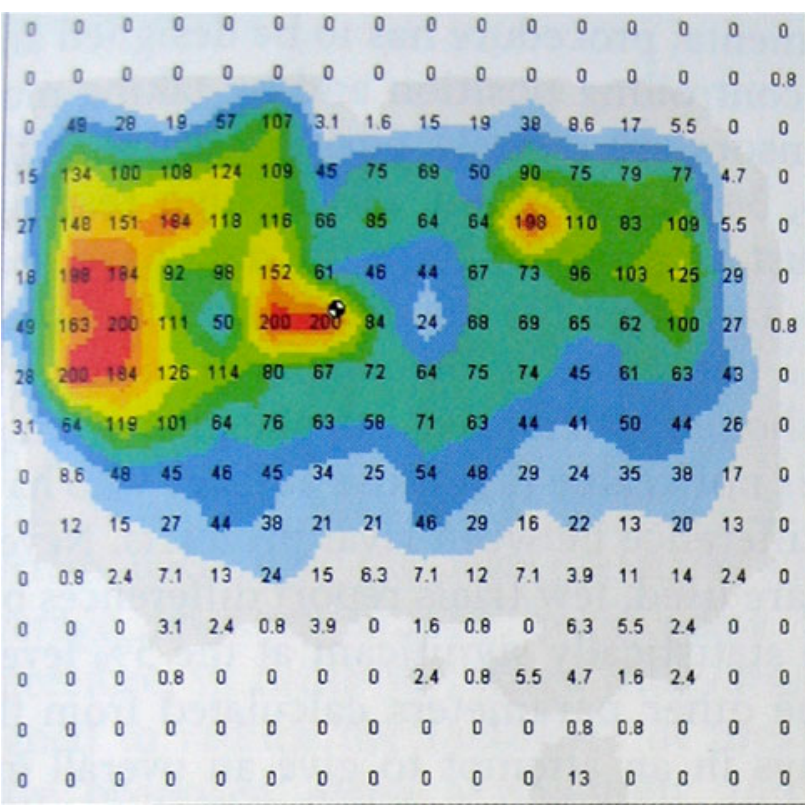

Fig. 1 Pressure distribution of a sitting person

disadvantage: the auditory signal is public. Other people can hear the signal as well, which compromises the privacy of the users. The user may maintain privacy by wearing a headset, but this might not only become uncomfortable after a certain period, but it may also inhibit communication with other people and media (e.g. television). Compared to visual signals, auditory signals convey less information, but speech would certainly be rich enough to inform the user about the current status.

Tactile feedback is also able to directly grab the attention of the users, but it can do it discreetly. Vibration motors that are attached to the body can be sensed immediately without alerting other or inhibiting the communication with others. It is therefore commonly used for silent alarms in mobile phones. A pilot study at Brunel University, tactile interfaces have been successfully used for visual impaired people and may offer advantages over auditory interfaces (Gustafson-Pearce et al. 2007). Because visual impaired people rely on auditory information from the environment, tactile feedback is a good choice for navigation since it does not interfere with auditory channel. It has also been shown that tactile feedback has been superior to visual feedback as a medium for warning car drivers of collisions (Scott and Gray 2007).

Tactile feedback seems particular successful in situations in which other modalities are already occupied. However, the amount of information that can be communicated is limited compared to visual and auditory communication. The main research question is therefore if tactile feedback can provide sufficient information to the user about critical postures. Before we describe the experimental setup to investigate this question, we will provide a short summary of the feedback system we developed.

\section{Design}

The design of our system consists of two main parts: a sensor subsystem and an actuator subsystem (see Fig. 2). The two subsystems currently communicate with each other through two wires, but in the future, we intend to use wireless communication. We will now describe the two subsystems in more detail.

\section{Sensing Subsystem}

The sensing sub system consists of 256 sensors that are connected into a force sensing array (FSA). This array is then connected to a microcontroller. We used Vista Medical's FSA Seating Assessment sensor mat (see Fig. 3) for an FSA. It is only $2 \mathrm{~mm}$ thick and measures $533 \mathrm{~mm}$ by $533 \mathrm{~mm}$ with an active sensing area of $430 \mathrm{~mm}$ by $430 \mathrm{~mm}$. This mat uses piezo resistive semi conductor polymers, that are sandwiched between two layers of highly conductive rip stop nylon fabric. The individual sensors are connected in a matrix that provides an array of

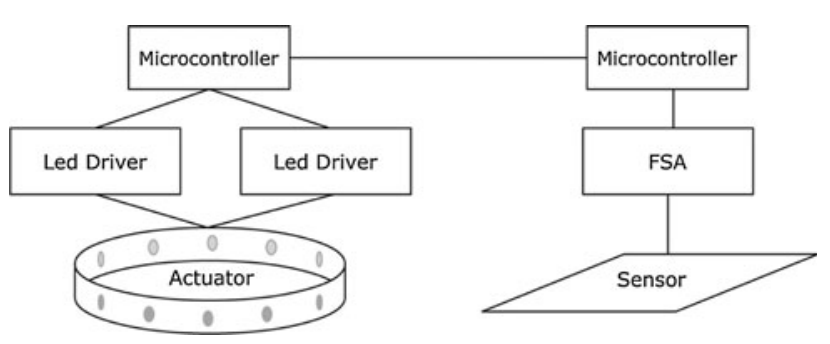

Fig. 2 The system architecture

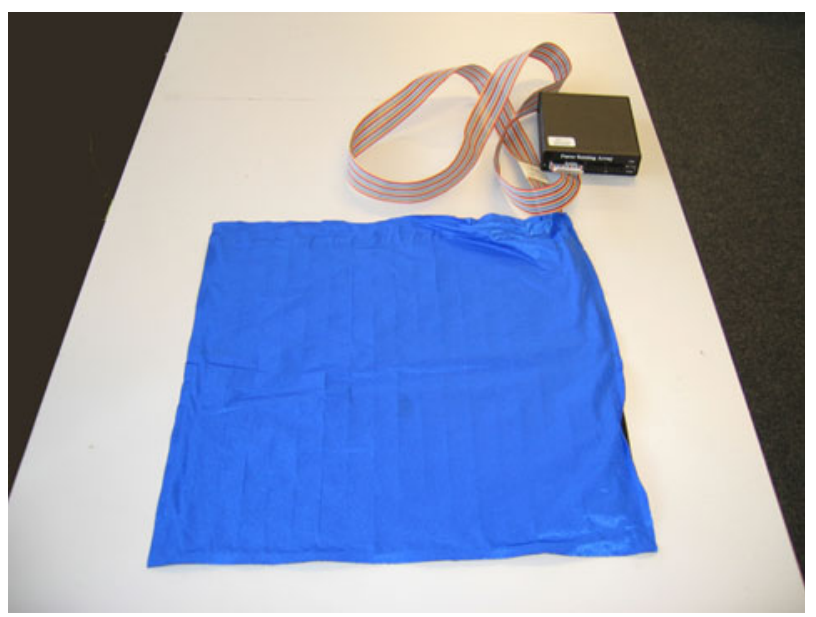

Fig. 3 Vista Medical's FSA Seating Assessment sensor mat 
force sensitive cells that measure the pressure distribution between the two surfaces. The cells are connected to an Arduino micro controller.

The 256 sensors provide a too detailed view on the pressure distribution on the mat. With only nine areas, it is possible to detect the nine standard postures (Hobson 1992) typically assumed by wheelchair users. The 256 sensors need to be mapped to nine areas. Since the anatomy of the users is different, it is necessary to adapt the mapping to each individual user (Fig. 4).

The sensing subsystem micro controller sends an alarm to the actuator subsystem micro controller if the pressure in an area exceeds a threshold. For each of the nine sections, the micro controller took four measurements per second. The number of sensors associated to a section varied. Section A might have 16 sensors, while section B might have only eight. Each area has a linear storage of 100 slots. This storage functions as a first-in-first-out storage register that provides a moving window of measurements.

If at least one sensor reading in an area was above the pressure threshold, a value of one was stored. If no sensor reading exceeded the pressure threshold, a zero was stored in the storage. Each area has its one storage and all of them used a first-in-first-out dropping policy. When value number 101 needs to be stored, the 1 st value gets dropped from the storage, and when value number 102 comes in, the 2nd value gets dropped, and so fourth. All values in the linear storage are summed and if the sum exceeded the time threshold of 20 , an alarm for the respective area was triggered.

Based on the feedback of several experts, we set the initial pressure threshold to $32 \mathrm{mmHg}$. The time threshold

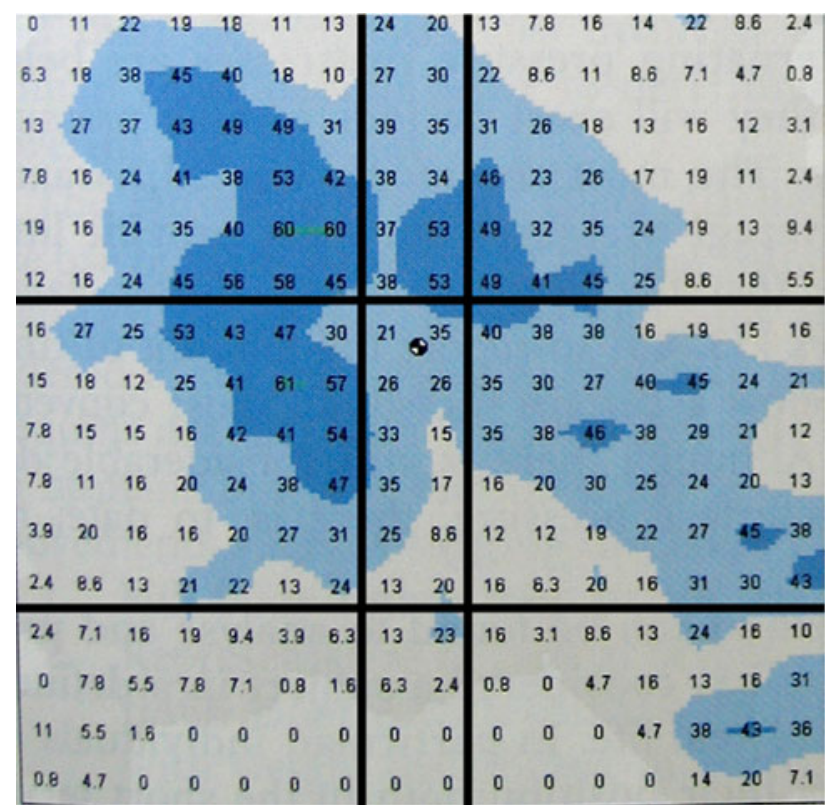

Fig. 4 An example mapping of the 256 sensors to nine areas was initially set to 20 , meaning that if the pressure threshold was exceeded for $12 \mathrm{~s}$ within $1 \mathrm{~min}$ in a certain section, the alarm was triggered. Both threshold must be considered preliminary and the system allows us to adjust them at any given time. Literature indicates that the combination of pressure and duration defines harmful postures. Reswick and Rogers (1975) proposed the curve shown in Fig. 5 to define harmful postures. Several studies indicated that this widely used benchmark might need to be updated (Gefen 2007; Linder Ganz et al. 2006). The threshold in our system can be adjusted as soon as improved guidelines have gained general acceptance.

\section{Actuating Subsystem}

The actuating subsystem consists of a $1 \mathrm{~m}$ belt to which 16 vibration motors are attached at a distance of $6 \mathrm{~cm}$ (see Fig. 7). Eight of these motors are attached to one LED driver (Maxim MAX7313) and eight to another. One MAX7313 has sixteen input/output ports and could in

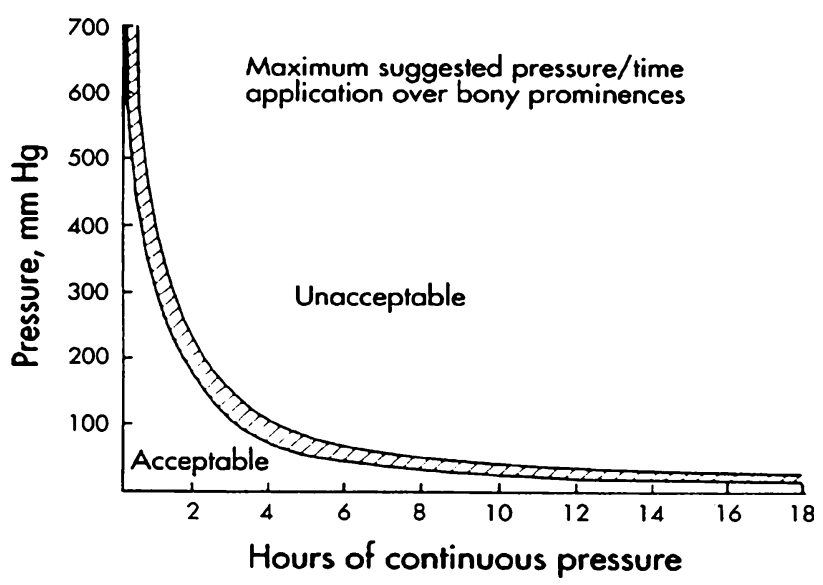

Fig. 5 Maximum suggested pressure over time (taken from Reswick and Rogers 1975)

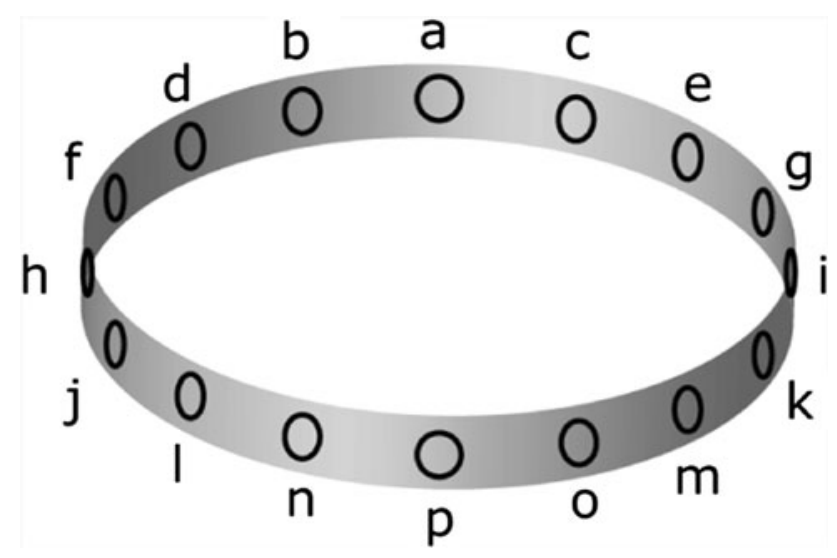

Fig. 6 Position of the motors on the belt 


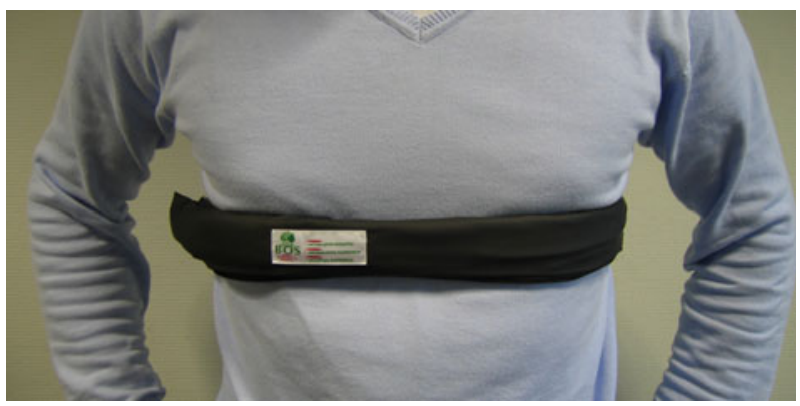

Fig. 7 Tactile feedback belt

principle drive the 16 vibration motors alone, but we decided to split the 16 motors over two LED drivers to reduce noise. The two LED drivers are then controlled through an Arduino micro controller using the $\mathrm{I}^{2} \mathrm{C}$ protocol. The vibration intensity of each motor could be controlled separately.

For the vibration motors we selected the MOT-0 motor from VOTI, which has a diameter of $10 \mathrm{~mm}$, a height of $3 \mathrm{~mm}$ and a weight of $1 \mathrm{~g}$. Three lithium coin cells ( 3 Volt each) powered the micro controller and the motors. Each motor requires 3 Volt and $70 \mathrm{~mA}$ and the micro controller requires 5 Volt. All the components have been integrated into the textile belt. The belt is worn around the chest and its width can be adjust with the Velcro closing strip. In summary, the belt can create vibration patter along the chest of the user.

\section{Evaluation of Feedback System}

The presence of several commercial products that map the pressure of the users indicates that this part of feedback systems is well understood and developed. We ourselves did not develop our own sensing mat, but used existing products. However, the part of communicating problematic postures to the user is less understood. We therefore conducted a preliminary experiment to determine if the preferable tactile feedback is able to communicate sufficiently well in comparison to auditory feedback. For practical reasons, we were not yet able to test the system with LSCI patients and used healthy subjects instead. We speculate that the sensory perception in the upper torso and cognitive processing of LSCI patients to approximate that of healthy people and therefore assume that a study with healthy subjects is able to provide initial insights into the feasibility of tactile feedback.

We performed a within participants experiment in which the type of feedback was the independent variable. It consisted of two conditions: tactile feedback or auditory feedback. The dependent variables were the reacting time and error of the user. To provide a realistic usage scenario we also included a secondary task since in normal life, the participants would also be busy with other tasks while they receive the posture feedback. The secondary task consisted of watching a silent cartoon movie on a computer screen. This visual distraction does not interfere with the auditory or tactile feedback. This visual task is an in-between step towards a rehabilitation game, that would most likely include a screen.

\section{Set Up}

The participant is positioned in a chair in front of the computer at a distance of $1 \mathrm{~m}$. A sensor mat was placed on the chair. The mat was connected to computer to automatically record the behavior of the user. The same computer also controlled the auditory or tactile feedback. The automatically created $\log$ file of this computer allowed us to deduct the reaction time and error of the users. In the auditory condition, the participants wore a headset to receive the feedback and in the tactile condition the participants were wearing the tactile feedback belt on their chest, right above their underwear.

The feedback the participants received were not the location of a problematic area, but instructions to what posture they should change to relief the problematic area. The audio feedback consisted of four verbal messages: forward, backward, left, right. The verbal messages were created using the TextAloud text-to-speech synthesizer. The length of the auditory messages ranged between 2.05 and $2.17 \mathrm{~s}$.

There are two types of vibrotactile feedback: impulse and continuous feedback (Lindeman et al. 2004). Impulse feedback refers to ballistic interaction, comparable to knocking. Continuous feedback refers to contact over a longer period of time. In this study, we decided to use continues feedback to indicate a direction towards which the user should be moving. The continues feedback describes the perceived sensation as one of a flow around the body. The vibration therefore mimics the irritation that healthy people would feel. Although the vibrations are not painful, we expected that the users naturally wanted to move away from them.

The tactile feedback consisted of four vibration patterns. The feedback used to instruct the user to move in a certain direction is listed in Table 1. Lets take the example of instructing the user to move forward. The motors are labeled according to the schema in Fig. 6. First the motor in the very back would start (a) before the its immediate neighboring motors $(b, c)$ to the right and left would start. The signal moved around the torso towards the front. Each motor would increase its intensity to a maximum, at which time the next motor would slowly start. The first motor then decreases its intensity back to zero (see Fig. 8 for the 
Table 1 Moving instructions (the letters refer to the motors in Figure 6)

\begin{tabular}{llllllllll}
\hline \multicolumn{7}{l}{ Activated vibration motor at time $t$} \\
\cline { 2 - 9 } & $t=0$ & $t=320 \mathrm{~ms}$ & $t=640 \mathrm{~ms}$ & $t=960 \mathrm{~ms}$ & $t=1280$ & $t=1600$ & $t=1920 \mathrm{~ms}$ & $t=2240 \mathrm{~ms}$ & $t=2560 \mathrm{~ms}$ \\
\hline Forward & $\mathrm{a}$ & $\mathrm{b}+\mathrm{c}$ & $\mathrm{d}+\mathrm{e}$ & $\mathrm{f}+\mathrm{g}$ & $\mathrm{h}+\mathrm{i}$ & $\mathrm{j}+\mathrm{k}$ & $1+\mathrm{m}$ & $\mathrm{n}+\mathrm{o}$ & $\mathrm{p}$ \\
Backward & $\mathrm{p}$ & $\mathrm{n}+\mathrm{o}$ & $1+\mathrm{m}$ & $\mathrm{j}+\mathrm{k}$ & $\mathrm{h}+\mathrm{i}$ & $\mathrm{f}+\mathrm{g}$ & $\mathrm{d}+\mathrm{e}$ & $\mathrm{b}+\mathrm{c}$ & $\mathrm{a}$ \\
Left & $\mathrm{h}$ & $\mathrm{f}+\mathrm{j}$ & $\mathrm{d}+1$ & $\mathrm{~b}+\mathrm{n}$ & $\mathrm{a}+\mathrm{p}$ & $\mathrm{c}+\mathrm{o}$ & $\mathrm{e}+\mathrm{m}$ & $\mathrm{g}+\mathrm{k}$ & $\mathrm{i}$ \\
Right & $\mathrm{i}$ & $\mathrm{g}+\mathrm{k}$ & $\mathrm{e}+\mathrm{m}$ & $\mathrm{c}+\mathrm{o}$ & $\mathrm{a}+\mathrm{p}$ & $\mathrm{b}+\mathrm{n}$ & $\mathrm{d}+1$ & $\mathrm{f}+\mathrm{j}$ & $\mathrm{h}$ \\
\hline
\end{tabular}

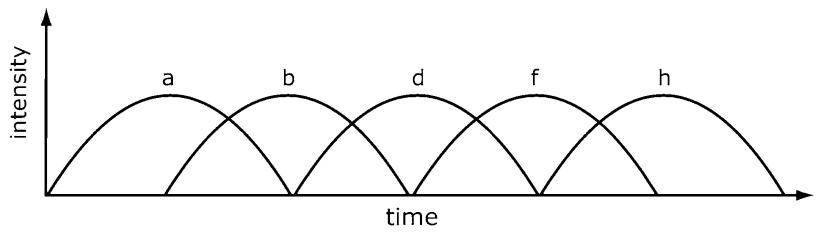

Fig. 8 Intensity over time of the moving forward pattern (right side only)

forward pattern for the right side). The complete interval of increasing and decreasing the vibration intensity was $640 \mathrm{~ms}$ for each motor. The complete duration for one vibration pattern was $3.2 \mathrm{~s}$.

\section{Participants}

Twenty six volunteers (22 men, 4 woman) from the Department Industrial Design at the Eindhoven University of Technology were recruited for the experiment. None of them were involved in the project or had previously joined related projects. Participants ranged in age from 21 to 27 years, with an average of 24 years. 12 participants were first randomly assigned to either the tactile condition and 14 to the audio condition.

\section{Measurements}

The computer automatically recorded the starting time of the signal, the time when the participant reached the new position and if the new position was the requested position. We could therefore calculate the reaction time and the sum or errors for each participant.

\section{Procedure}

After welcoming the participants, the experimenter guided the participants to the chair in which they signed a written consent form. During this time, the experimenter checked if the participant is in a central position on the chair and whether the participant has any items on them that could influence the measurements, such as wallets in their back pockets or buttons stitched onto the trousers.
The participants were instructed to sit in the center of the chair. They were then asked to either wear the headphone or the belt. Next, they were instructed to watch the cartoon. Once they would receive the signal (auditory or tactile) they would have to move to the indicated position as quickly as possible. Afterwards they should move back to the center position. After a short training session, the first session of the experiment started. The participants received twelve signals to which they had to react. The order of the signals was randomized. To avoid a training effect, the time between signals was also randomized, ranging between 5 and $20 \mathrm{~s}$. The participants were not notified if their change of posture was correct or not.

Afterwards, the participant would use the other feedback device and again receive instructions. They then had the opportunity to try out the system in a short training session. Afterwards the second session of the experiment started that the same structure as the first session. After the second session, the participants were debriefed. The order of the two sessions was cross-balanced across the participants.

\section{Results}

We performed a repeated measure analysis of variance (ANOVA) in which the feedback type (auditory or tactile) was the independent variable and the reaction time and error were the dependent variables. We did not find an order effect between the two experimental conditions. Levene's test of equality of error variance revealed that the variances for the dependent variables were equally distributed. The feedback type had a significant $(F(1,23)=$ 6.964, $p=0.015)$ effect on reaction time, but it did not have a significant effect on error $(F(1,23)=1.944, p=$ 0.177 ; Table 2.

Table 2 Reaction time (in seconds) and error counts

\begin{tabular}{llllll}
\hline & \multicolumn{2}{l}{ Reaction time } & & \multicolumn{2}{l}{ Error } \\
\cline { 2 - 3 } & Mean & SD & & Mean & SD \\
\hline Tactile & 2.4908 & 0.30134 & & 0.5833 & 0.66856 \\
Auditory & 2.0707 & 0.39566 & & 0.2143 & 0.42582 \\
\hline
\end{tabular}




\section{Conclusions}

Previous studies indicated that in particular peak pressures and a lack of posture activity promote Decubitus ulcers (Gefen 2007; Hobson 1992; Linder-Ganz et al. 2007). We presented a system that is able to detect harmful posture of users and provides discreet tactile instructions to what posture the user should change. By guiding the users into new postures, the system is hopefully able to reduce the risk of developing Decubitus ulcers.

The results of the experiment show that tactile feedback is an appropriate medium for posture feedback. Although the participants were slightly slower in moving to the indicated posture, the difference is so small that it can be neglected. We speculate about two reasons for the small difference. The first reason is that the tactile signal itself took on average half a second longer to be produced than the audio command. A second reason might be that the novelty of the tactile feedback. We speculate that it might take longer for the user to get used to tactile feedback than this current study allowed for. In any case, the fraction of a second difference is unlikely to have an impact on the overall Decubitus ulcers prevention. It is also possible that an impulse tactile feedback might speed up the recognition process and enable to the users to react as quickly to the tactile feedback as to the auditory feedback.

The next step for this project is to study the system over a longer period in the context of use. In our current study, the participants used the system only for a short period of time, and we were obviously not able to detect problematic postures within the framework of several hours. Furthermore, we want to test the system with LSCI patients to verify that their response do not deviate too much from our results. In the end, LSCI patients are an important target group and hence they are the true benchmark.

We also want to further explore the opportunities of the system for rehabilitation games. Due to the discreet nature of tactile feedback, such a game would not be limited to a specific rehabilitation session, but it could be played throughout the day. The system could also be used as a generic game controller, allowing SCI patients to even play Dance Dance Revolution. The results from out study indicate that our system is a suitable candidate for game play.

We need to clearly state that we did not perform a clinical study that could justify a claim that our system makes a contribution to the prevention of Decubitus ulcers. We could only show that tactile feedback is viable option to auditory feedback to communicate feedback about the posture of the user. An extended clinical study would be necessary before a system as described above could be admitted into the market for medical products.
Open Access This article is distributed under the terms of the Creative Commons Attribution Noncommercial License which permits any noncommercial use, distribution, and reproduction in any medium, provided the original author(s) and source are credited.

\section{References}

Braden, B., \& Bergstrom, N. (1987). A conceptual schema for the study of the etiology of pressure sores. Rehabilitation Nursing, $12(1), 8-12$.

Braden, B., \& Maklebust, J. (2005). Preventing pressure ulcers with the Braden scale: An update on this easy-to-use tool that assesses a patient's risk. American Journal of Nursing, 105(6), 70-72.

Health Council of The Netherlands. (1999). Pressure ulcers (No. 1999/23). The Hague: Health Council of The Netherlands.

Gefen, A. (2007). The biomechanics of sitting-acquired pressure ulcers in patients with spinal cord injury lesions. International Wound Journal, 4(3), 222-231. doi:10.1111/j.1742-481X.2007. 00330.x.

Goldberg, G., Rubinsky, H., Irvin, E., Linneman, E., Knapke, J., \& Ryan, M. (2008). Doing WiiHab: Experience with the Wii video game system in acquired brain injury rehabilitation: 0034. The Journal of Head Trauma Rehabilitation, 23(5), 350.

Gustafson-Pearce, O., Billett, E., \& Cecelja, F. (2007). Comparison between audio and tactile systems for delivering simple navigational information to visually impaired pedestrians. British Journal of Visual Impairment, 25(3), 255-265. doi:10.1177/026461960 7079807.

Harvin, J., \& Hargest, T. (1970). The air-fluidized bed: A new concept in the treatment of decubitus ulcers. Critical care nursing clinics of North America, 5(1), 181-187.

Hobson, D. A. (1992). Comparative effects of posture on pressure and shear at the body-seat interface. Journal of Rehabilitation Research and Development, 29(4), 21-31.

Leder, R. S., Azcarate, G., Savage, R., Savage, S., Sucar, L. E., \& Reinkensmeyer, D., et al. (2008). Nintendo Wii remote for computer simulated arm and wrist therapy in stroke survivors with upper extremity hemipariesis. Proceedings of the Virtual Rehabilitation, Vancouver pp. 74-74. doi:10.1109/ICVR.2008. 4625137.

Lindeman, R. W., Page, R., Yanagida, Y., \& Sibert, J. L. (2004). Towards full-body haptic feedback: The design and deployment of a spatialized vibrotactile feedback system. Proceedings of the Proceedings of the ACM symposium on Virtual reality software and technology, Hong Kong pp. 146-149. doi:10.1145/1077534. 1077562

Linder Ganz, E., Engelberg, S., Scheinowitz, M., \& Gefen, A. (2006). Pressure-time cell death threshold for albino rat skeletal muscles as related to pressure sore biomechanics. Journal of Biomechanics, 39(14), 2725-2732. doi:10.1016/j.jbiomech.2005.08.010.

Linder-Ganz, E., Scheinowitz, M., Yizhar, Z., Margulies, S. S., \& Gefen, A. (2007). How do normals move during prolonged wheelchair-sitting? Technology and Health Care, 15(3), 195-202.

Merbitz, C. J., King, R. B., Bleiberg, J., \& Grip, J. C. (1985). Wheelchair push-ups: Measuring pressure relief frequency. Archives of Physical Medicine and Rehabilitation, 66(7), 433438.

Reswick, J., \& Rogers, J. (1975). Experience at Rancho Los Amigos Hospital with devices and techniques to prevent pressure sores in bedsore biomechanics. In R. M. Kenedi, J. M. Cowden, \& J. T. Scales (Eds.), Bedsore biomechanics: Proceedings of a seminar on tissue viability and clinical applications. Baltimore: University Park Press. 
Revalidatiefonds. (2009). Zitdrukverlaging bij mensen met een dwarslaesie Retrieved February 17th, 2009, from http://www. revalidatiefonds.nl/content/view/83/77/.

Roemer, R., Meisel, H., \& Parrish, W. (1975). Automated bed to aid pulmonary drainage and prevent decubitus ulcers. Medical and Biological Engineering and Computing, 13(1), 78-83. doi: 10.1007/BF02478191.

Scott, J. J., \& Gray, R. (2007). Comparison of driver brake reaction times to multimodal rear-end collision warnings. Proceedings of the Fourth International Driving Symposium on Human Factors in Driver Assessment, Training and Vehicle Design, Stevenson pp. 285-291.
Sprigle, S., \& Schuch, J. (1993). Using seat contour during seating evaluations of individuals with SCI. Assistive Technology, 5(1), 24-35.

Stockton, L., \& Parker, D. (2002). Pressure relief behaviour and the prevention of pressure ulcers in wheelchair users in the community. Journal of tissue viability, 12(3), 88-90.

Strauss, M. J., Gong, J., Gary, B. D., Kalsbeek, W. D., \& Spear, S. (1991). The cost of home air-fluidized therapy for pressure sores: A randomized controlled trial. Journal of Family Practice, 33(1), $52-59$. 